\title{
Bienestar Subjetivo y Satisfacción Vital del Profesorado
}

\author{
Teachers' Subjective Well-being and Satisfaction with Life
}

\author{
Eva Ma Muñoz Campos * \\ Antonio Fernández González \\ Liliana Jacott
}

Universidad Autónoma de Madrid, España

\begin{abstract}
El bienestar subjetivo como aspecto indispensable de la calidad de vida, ha sido objeto de estudio durante las últimas décadas. Dentro del contexto escolar esta inquietud se ha extendido a la necesidad de investigar estos aspectos en dicho ámbito, específicamente en la población docente. El objetivo de esta investigación es analizar el bienestar subjetivo y el grado de Satisfacción Vital de los profesionales que se dedican a la docencia y la influencia que tienen sobre estos factores distintas variables asociadas a elementos sociodemográficos y aspectos relacionados con el desempeño de la labor docente. Estas medidas de bienestar se han recogido de un total de 180 docentes de la Comunidad de Madrid que desempeñan su labor profesional en centros de titularidad pública, privada y concertada en distintas etapas educativas. Para la consecución del objetivo de la investigación se aplicaron dos escalas, la Escala de Bienestar Psicológico para Adultos (Casullo y Brenlla, 2002) y la Escala de Satisfacción con la Vida (Diener et al., 1985) y un cuestionario de recogida de datos sociodemográficos. Los resultados muestran que existen correlaciones significativas con respecto a la satisfacción vital y el bienestar subjetivo en las variables de género, edad, titularidad del centro, etapa educativa y años de experiencia docente, resaltando la importancia de aumentar en número la muestra en futuras investigaciones para profundizar en el estudio del Bienestar subjetivo y la Satisfacción Vital del profesorado.
\end{abstract}

Descriptores: Calidad de vida laboral; Enseñanza; Satisfacción en el trabajo; Docente; Escuela.

Subjective wellbeing as an indispensable aspect of the quality of life, has been the object of study during the last decades. Within the school context this concern has been extended to the need to investigate these aspects in the school environment, specifically in the teaching population. The objective of this research is to analyze the subjective wellbeing and the Satisfaction with life of the professionals who dedicate themselves to teaching and the influence that these variables have on different aspects associated with sociodemographic elements and related to the teaching profession. These welfare measures have been collected from a total of 180 teachers of the Community of Madrid who perform their professional work in community and private centers in different educational stages. To achieve the objective of the research, two scales of measurement were applied, the Psychological Wellness Scale for Adults (Casullo y Brenlla, 2002) and the Satisfaction with Life Scale (Diener et al., 1985) and a questionnaire of sociodemographic data. The results show that there are significant correlations between the Satisfaction with life and Subjective Wellbeing in the variables of gender, age, ownership of the center, educational stage and years of teaching experience, highlighting the importance of increasing the number of the sample in future research for deepen in the study of subjective well-being and satisfaction with life of the teaching staff.

Keywords: Quality of working life; Teaching; Job satisfaction; Teachers; Schools.

*Contacto: eva.munnoz@uam.es

ISSN: $1696-4713$

www.rinace.net/reice/

revistas.uam.es/reice
Recibido: 1 de septiembre 2017

$1^{\text {a }}$ Evaluación: 23 de octubre 2017

$2^{\text {a }}$ Evaluación: 9 de noviembre 2017

Aceptado: $\quad 15$ de diciembre 2017 


\section{Revisión de la literatura}

La felicidad, la satisfacción y el bienestar generan un interés creciente en los últimos años en diversos ámbitos: económico, social y científico. Esta inquietud se extiende hacia la necesidad de investigar estos aspectos en el ámbito escolar incorporando los hallazgos de la Psicología Positiva y asumiendo un enfoque positivo del desarrollo de la personalidad y del funcionamiento humano óptimo.

Los conceptos de bienestar, calidad de vida y satisfacción han sido objeto de múltiples investigaciones (Diener y Seligman, 2004; Extremera, Salguero y Fernández-Berrocal, 2011; Keyes, Shmotkin y Ryff, 2002). Si bien inicialmente la psicología puso el énfasis en el estudio de la patología, contribuyendo con aportes muy valiosos en el campo científico, no obstante, puede decirse que estos resultaban incompletos si tenemos en cuenta una concepción más holística e integradora del ser humano. En esa circunstancia aparece la Psicología Positiva (Csikszentmihalyi y Seligman, 2000) conceptualizada como ciencia aplicada al conocimiento y desarrollo de las fortalezas y virtudes humanas, promoviendo la felicidad para la construcción y el enriquecimiento integral de individuos y comunidades. Una de sus premisas principales consiste en abordar la enfermedad de manera compensatoria entendiendo que muchos de los aspectos que influyen en la adquisición de un comportamiento resiliente pueden ser aprendidos.

Aunque son varias y muy diversas las definiciones de bienestar y satisfacción, estas poseen elementos comunes entre los que podríamos destacar los siguientes: estado de satisfacción personal, de comodidad y de confort, considerando como positivos aspectos tales como la salud, el éxito social, económico y profesional y la armonía consigo mismo y con el entorno. En base a ello podemos concluir que, cuando la persona experimenta satisfacción con su vida, si frecuentemente su estado anímico es bueno y sólo ocasionalmente experimenta emociones poco placenteras como tristeza o rabia, experimenta bienestar (Andrews y Withey, 1976; Campbell, Converse y Rodgers, 1976; Veenhoven, 1991).

En lo que se refiere al contexto escolar, tal como algunos autores señalan, el bienestar docente constituye una corriente de investigación alternativa que surge en contraposición a los estudios previos que estaban más centrados en analizar el malestar de los docentes, teniendo como foco sus experiencias negativas (De Pablos, Bravo y González, 2011). Resulta interesante destacar cómo si bien inicialmente las líneas de investigación en este ámbito se abordaron fundamentalmente desde una perspectiva más psicológica y no tanto educativa, no obstante, en las últimas décadas distintos autores ponen de manifiesto su interés por abordar el bienestar emocional del profesorado (Verhoeven et al., 2003).

La satisfacción con la vida se entiende como la evaluación global que las personas realizan sobre su calidad de vida bien ante circunstancias concretas o bien ante ámbitos concretos como la familia, los amigos, entre otros (Seligson, Huebner y Valois, 2003). El componente cognitivo de la satisfacción hace referencia al bienestar subjetivo (Andrews y Withey, 1976; Diener et al., 1985) entendiendo éste como el grado en que una persona evalúa la calidad global de su vida en conjunto de forma positiva (Veenhoven, 1991). El estudio de la satisfacción con la vida adquiere especial relevancia puesto que los datos obtenidos con estas investigaciones nos permitirán prevenir, detectar e intervenir en poblaciones de riesgo social (Seligson, Huebner y Valois, 2003).

El concepto de bienestar psicológico ha generado controversias dentro del campo de la psicología por diversas razones entre las que cabe destacar el uso generalizado del término 
bienestar como sinónimo de calidad de vida, felicidad, funcionamiento positivo, resiliencia y motivación cuando distan de tener el mismo significado (Ballesteros, Medina y Caycedo, 2006). Por una parte, tal como señalan Casas, Roscih y Alsinet (2000), los trabajos pioneros de Bradburn y su equipo que desarrollaron la Escala ABS o Escala de Balance Afectivo (Affect Balance Scale), demostraron que la felicidad humana era una cuestión que podía ser abordada desde una perspectiva científica. Al mismo tiempo que aportaron evidencia suficiente para apoyar un hecho que entonces resultaba sorprendente referido a que los afectos positivos no correlacionan siempre con los afectos negativos, aspecto que ha pasado a formar parte de todos los modelos de estudio de la calidad de vida. Y paralelamente, los trabajos de Cantril con la Escala SASS (Self-Anchoring Striving Scale), demostraron que era posible estudiar rigurosamente la satisfacción con nuestras vidas, bien globalmente, o en ámbitos concretos de las mismas. Estas dos visiones han dado lugar a lo que en el ámbito científico se ha denominado la aproximación felicidad versus la aproximación satisfacción.

El bienestar subjetivo ha sido investigado desde tres modelos teóricos (Eid y Larsen, 2008): el modelo ambientalista, el modelo psicológico y por último el modelo interaccionista. El primer modelo defiende que el bienestar subjetivo depende de las vinculaciones externas y las características del entorno, en términos de bienestar docente éste estaría condicionado por el contexto educativo y variables socioculturales, oponiéndose a esta teoría, el modelo psicológico propone que el bienestar depende de las características personales del sujeto, siendo muchos y variados los estudios que han tratado de identificar las variables de personalidad que correlacionan de manera positiva con el bienestar subjetivo (Harris y Lightsey, 2005). Finalmente, el modelo interaccionista propone que el bienestar es el fruto de la interacción de los factores mencionados en las dos teorías anteriores.

El bienestar psicológico subjetivo (BPS) es concebido como resultado de la integración de dos tradiciones conceptuales: una tradición de tendencia hedónica referida al bienestar subjetivo y otra de tendencia eudamónica referida al bienestar psicológico ligada al desarrollo del potencial humano (Díaz et al., 2006). El bienestar subjetivo, está centrado en los aspectos afectivos-emocionales, haciendo referencia a los estados de ánimo del sujeto y a cómo y porqué las personas experimentan su vida de forma positiva (Cornejo y Quiñónez, 2007). Y el bienestar psicológico, centrado en los aspectos cognitivos del bienestar, haciendo referencia a la evaluación de la satisfacción que hacen los sujetos de su propia vida incluyendo aquellos elementos relativos al desarrollo personal, estilo y manera de afrontar los retos y persistencia en la consecución de metas personales (Vázquez et al., 2009).

En la actualidad se reconoce que ambas perspectivas son distintas (Diener y Ryan, 2009) pero complementarias (Ryan y Deci, 2001) y estas conclusiones han dado lugar a nuevas líneas de investigación entre las que destaca la de Seligman (2011) que propuso un abordaje integrado del bienestar psicológico en su primera teoría que denominó Felicidad auténtica (Authentic Happiness Theory) y que recientemente ha reformulado convirtiéndola en la Teoría del bienestar bajo el acrónimo PERMA (possitive emotions, engagement, relationships and meaning).

Los estudios sobre bienestar docente aumentaron considerablemente en número debido principalmente a los notables cambios que en las últimas décadas ha experimentado este sector (p.ej., precariedad laboral, masificación en las aulas, continuo cambio en las políticas 
educativas, etc.). Y junto a ello, cabe señalar también el significativo aumento de estudios e investigaciones con respecto al malestar entre los docentes (Chávez, 2009). En esta línea de investigación se han desarrollado varios modelos, como el modelo trifactorial de Maslach (2003) en el que se analiza el síndrome burnout del profesorado agrupando la sintomatología de este síndrome psicológico en tres bloques: sensación de bajo logro, despersonalización y agotamiento emocional. Otro de los modelos es el denominado modelo de demanda-control-apoyo social o modelo de Karasek, siendo éste el más utilizado en el estudio de los factores psicosociales que influyen en el desempeño. El planteamiento es que a mayor nivel de demanda laboral y menor grado de control sobre el trabajo, los niveles de malestar aumentan y los niveles de satisfacción laboral disminuyen (Karasek y Theorell, 1990).

Por el contrario, si nos centramos en el bienestar docente (Carrasco y Bernal, 2008; Marchesi, 2007), las observaciones realizadas en las últimas investigaciones nos indican que el ejercicio de la actividad docente puede llegar a desencadenar, o bien sensación de abatimiento y desánimo o bien lo contrario, motivación e ilusión. Según las investigaciones de Hulin y Judge (2003) los aspectos vinculados a la satisfacción laboral tienen tres componentes diferenciados: un componente cognitivo (evaluativo), otro afectivo (emocional) y otro conductual (comportamental). Siguiendo esta línea de investigación y teniendo en cuenta la dimensión afectiva y emocional de la satisfacción y el bienestar en el ámbito laboral, en este estudio hemos decidido llevar a cabo una medición del bienestar psicológico del profesorado y su satisfacción vital.

En el presente estudio se analiza el grado de satisfacción vital y el bienestar subjetivo de los profesionales que se dedican a la docencia y se establece cuál es la relación de ambos constructos con las variables de género, edad, etapa educativa en la que realizan su desempeño profesional y años de experiencia docente, teniendo en cuenta que existe numerosa evidencia que apoya la existencia de una relación entre condiciones de trabajo y salud laboral, productividad y permanencia en el empleo.

Nuestra hipótesis de trabajo parte de que las variables demográficas de edad, años de experiencia docente, titularidad del centro y etapa educativa en la que desempeñan su tarea como docentes influyen en el bienestar subjetivo y la satisfacción vital del profesorado.

\section{Método}

\section{Participantes}

Los participantes son docentes de la Comunidad de Madrid (180); 125 mujeres (69,4\%), edad media de 40,6 años y 55 hombres (30,6\%), edad media de 40,8. Dichas edades estaban comprendidas entre los 22 y los 68 años: 25 participantes con edades entre los 20 y 30; 63 entre los 31 y 40; 62 entre los 41 y 50; 26 entre los 51 y los 60, y 4 mayores de 61 años. Respecto a las etapas educativas, 9 son profesores de Universidad; 28 de Bachillerato; 50 de Educación Secundaria Obligatoria; 19 de Formación Profesional; 53 de Educación Infantil y Primaria y 20 de Educación Especial. La experiencia docente media son 13,2 años (84, el 46,7\%, hasta 10 años; 63 , el 35\%, entre 10 y 20 años y 33 , el 18,3\%, más de 20 años). Finalmente, el $67 \%$ de los docentes prestan sus servicios en centros de titularidad pública, el $29 \%$ en privados-concertados y el $4 \%$ en privados.

\section{Instrumentos}


Para la realización del estudio se utilizaron dos instrumentos y un cuestionario que se describen a continuación:

The Satisfaction with Life Scale-SWLS (Diener et al., 1985). Es una escala multi-ítem que evalúa la satisfacción con la vida como un proceso de juicio cognitivo. Se utilizó la adaptación española (Atienza et al., 2000) en su versión reducida con ítems como: "Hasta ahora he conseguido de la vida las cosas que considero importantes", etc. Las opciones de respuesta se presentan en una escala tipo Likert de 5 puntos, oscilando entre Muy en desacuerdo (respuesta a la que se le asigna 1 punto) y Muy de acuerdo (respuesta a la que se le asignan 5 puntos) señalando así baja y alta satisfacción. La puntuación máxima es de 25 puntos y la mínima de 5. Pavot y Diener (1993) propusieron una serie de criterios para clasificar a las personas con respecto a una serie de puntos de corte que permite la interpretación de los resultados obtenidos de la siguiente manera: 25-21 muy satisfecho, 20-16 satisfecho, 15 neutro, 14-11 insatisfecho y de 10-5 muy insatisfecho. Muestra una alta consistencia interna con valores de $\alpha$ que oscilan entre 0,79 y 0,89 garantizando su fiabilidad. La escala ofrece la posibilidad de establecer cuáles son las distintas vías que nos permiten acceder al bienestar.

Escala de Bienestar Psicológico para Adultos-BIEPS-A (Casullo y Brenlla, 2002). Evalúa el bienestar psicológico (Ryff, 1989; Ryff y Singer, 1996). La escala está compuesta por 13 ítems, los cuales pueden aplicarse en forma individual o grupal, de forma autoadministrada. Los sujetos deben leer cada ítem y responder sobre lo que sintieron y pensaron durante el último mes. Las alternativas de respuesta son: de acuerdo (3 puntos), ni de acuerdo ni en desacuerdo (2 puntos), y en desacuerdo ( 1 punto).

- Control de situaciones. Las puntuaciones altas indican que la persona tiene sensación de control y auto-competencia, puede crear o manipular contextos para adecuarlos a sus propias necesidades e intereses. En cambio, aquellos que presentan una baja sensación de control tienen dificultades para manejar los asuntos de la vida diaria, no se dan cuenta de las oportunidades y creen que son incapaces de modificar el ambiente.

- Aceptación de sí mismo. Puede aceptar los múltiples aspectos de sí mismo, incluyendo los buenos y los malos. Se siente bien acerca del pasado. Si el sujeto obtiene un bajo puntaje, indica que está desilusionado respecto de su vida pasada, le gustaría ser diferente de cómo es y se siente insatisfecho consigo mismo.

- Vínculos psicosociales. Si posee una puntuación alta, el sujeto es cálido, confía en los demás, puede establecer buenos vínculos, tiene capacidad de empatía y es afectivo. En caso contrario, tiene pocas relaciones con los demás, es aislado, se siente frustrado en los vínculos que establece y no puede mantener compromisos con los demás.

- Autonomía. Cuando puntúa alto, el sujeto puede tomar decisiones de modo independiente, es asertivo y confía en su propio juicio. Las puntuaciones bajas indican que el individuo es emocionalmente inestable, depende de los demás para tomar decisiones y le preocupa lo que piensan los otros.

- Proyectos. Indica que tiene metas y proyectos en la vida, considera que la vida tiene significado y posee valores que hacen que su vida tenga sentido. Las puntuaciones bajas indican que la vida carece de sentido y de significado, el sujeto tiene pocas metas y proyectos y no puede establecer propósitos. 
- Interpretación de la escala. En puntuaciones inferiores al percentil $25 \mathrm{se}$ considera que la persona posee un bajo nivel de bienestar psicológico en varias de sus áreas vitales. Un nivel de bienestar psicológico promedio se observa en puntuaciones mayores al percentil 50, indicando que el sujeto se siente satisfecho con distintos aspectos de su vida.

Los ítems de la escala están redactados en formato directo, obteniéndose la puntuación total de la suma de las puntuaciones asignadas a cada ítem. La escala no admite respuestas en blanco. La puntuación global directa posteriormente se transforma a percentiles. Las puntuaciones correspondientes al percentil 95 indican un alto nivel de bienestar, señalando que el sujeto se siente muy satisfecho con su vida en general. La validez y fiabilidad de la escala ha quedado probada en distintos estudios (Casullo y Solano, 2000; Domínguez, 2014).

Cuestionario de variables sociodemográficas: El cuestionario recogió información de variables sociodemográficas como la edad, el género, los años de experiencia docente, la etapa educativa en la que imparten docencia y titularidad del centro en el que desempeñan su actividad profesional.

\section{Procedimiento}

El diseño es el propio de una investigación cuantitativa y una metodología ex post facto. Dada la naturaleza de la investigación y su realización en el ámbito educativo, la muestra se compone exclusivamente de docentes seleccionados a través de un muestreo probabilístico estratificado simple. Se pidió la participación a docentes de diversas etapas educativas de la Comunidad de Madrid que aceptaron formar parte de la investigación voluntariamente. Participar en la investigación no proporcionó ningún tipo de gratificación en horario lectivo a los docentes y se garantizó la confidencialidad de los datos recogidos. Se aplicó en primer lugar el cuestionario sociodemográfico, en segundo lugar, la escala BIEPS-A y por último la escala SWLS. La aplicación de los tres instrumentos de recogida de datos se llevó a cabo en una única sesión. Para analizar los datos se utilizó el programa SPSS versión 22 para Windows 2013.

\section{Resultados}

Los resultados que se exponen a continuación nos ofrecen el impacto que tienen las distintas variables estudiadas en el cuestionario demográfico con respecto a la satisfacción vital y el bienestar subjetivo de los profesionales que se dedican a la docencia.

En el cuadro 1 se muestran los resultados de índices por variables, en la que podemos observar que con respecto a la edad la franja que muestra más satisfacción vital y bienestar es en aquellos sujetos de 61 años o más. Con respecto al género las mujeres revelan mayor satisfacción mientras que los resultados recogen mayor bienestar en los hombres. Si tenemos en cuenta los años de experiencia docente el mayor índice de bienestar aparece en aquellos profesionales que cuentan entre 11 y 21 años de experiencia docente, encontrando los mismos resultados con respecto al índice de bienestar. La etapa educativa en la que los participantes mostraron una mayor satisfacción y bienestar fue la universitaria y con respecto a la titularidad del centro en el que imparten docencia, los participantes que mostraron un mayor índice de bienestar y satisfacción fueron aquellos 
que trabajan en centros de titularidad pública y los que menos en centros de titularidad privada.

Cuadro 1. Resultado de índices por variables

\begin{tabular}{|c|c|c|c|}
\hline & & $\begin{array}{c}\text { ÍNDICE } \\
\text { SATISFACCIÓN* }\end{array}$ & $\begin{array}{c}\text { ÍNDICE } \\
\text { BIENESTAR* }\end{array}$ \\
\hline \multirow{5}{*}{ Edad } & De 20 a 30 años & 3,60 & 2,55 \\
\hline & De 31 a 40 años & 3,80 & 2,48 \\
\hline & De 41 a 50 años & 3,93 & 2,62 \\
\hline & De 51 a 60 años & 3,72 & 2,55 \\
\hline & De 61 o más años & 4,65 & 2,64 \\
\hline \multirow{2}{*}{ Género } & Hombre & 3,80 & 2,58 \\
\hline & Mujer & 3,84 & 2,54 \\
\hline \multirow{3}{*}{$\begin{array}{l}\text { Años de } \\
\text { experiencia } \\
\text { docente }\end{array}$} & De 1 a 10 años & 3,77 & 2,51 \\
\hline & De 11 a 20 años & 3,92 & 2,62 \\
\hline & 21 o más años & 3,79 & 2,54 \\
\hline \multirow{6}{*}{$\begin{array}{l}\text { Etapa en la que } \\
\text { impartes docencia }\end{array}$} & Bachillerato & 3,96 & 2,55 \\
\hline & Colegio de Educación Especial & 3,81 & 2,56 \\
\hline & E.S.O. & 3,66 & 2,52 \\
\hline & Educación Infantil y Primaria & 3,79 & 2,54 \\
\hline & F.P. & 3,99 & 2,56 \\
\hline & Universidad & 4,07 & 2,69 \\
\hline \multirow{3}{*}{$\begin{array}{l}\text { Titularidad del } \\
\text { Colegio/IES }\end{array}$} & Privada & 3,20 & 2,41 \\
\hline & Privada/Concertada & 3,81 & 2,57 \\
\hline & Pública & 3,87 & 2,56 \\
\hline
\end{tabular}

Nota: * Valor de la Media.

Fuente: Elaboración propia.

Para analizar los datos referentes a la Satisfacción vital se procedió a realizar un análisis descriptivo de cada uno de los ítems que conforman la Escala SWLS. En función de los resultados obtenidos, el $34 \%$ de los participantes se encuentran muy satisfechos con su vida, el $51 \%$ satisfechos, el 5\% encuentran su grado de satisfacción vital de manera neutra, el $8 \%$ se encuentran insatisfechos y un $2 \%$ muy insatisfechos. Como se puede apreciar en el cuadro 2 el aspecto que demuestra mayor satisfacción es el recogido por el ítem "Hasta ahora he conseguido de la vida las cosas que considero importantes" y el aspecto en el que los participantes puntuaron más bajo con respecto a su satisfacción vital fue "Si pudiera vivir mi vida otra vez no cambiaría casi nada”.

Con respecto a los resultados obtenidos de la escala de Bienestar, se realizó un estudio de la media y la desviación típica para cada uno de los ítems que conforman la escala BIEPSA cuyos resultados quedan reflejados en el cuadro 3. De la descripción de los datos obtenidos podemos destacar que los aspectos que reflejan mayor bienestar para los participantes del estudio fueron: "Creo que en general me llevo bien con la gente" y "Cuento con personas que me ayudan si lo necesito", mientras que el aspecto menos elegido fue "Puedo tomar decisiones sin dudar mucho" y "En general hago lo que quiero, soy poco influenciable". 
Cuadro 2. Estadísticos descriptivos escala SWLS

\begin{tabular}{lcc}
\hline & M & DT \\
\hline En la mayoría de los aspectos mi vida es como yo quiero que sea. & 3,76 & 0,802 \\
Las circunstancias de mi vida son muy buenas. & 3,81 & 0,806 \\
Estoy satisfecho con mi vida. & 3,93 & 0,800 \\
Hasta ahora he conseguido de la vida las cosas que considero importantes. & 4,08 & 0,836 \\
Si pudiera vivir mi vida otra vez no cambiaría casi nada. & 3,56 & 1,074 \\
\hline
\end{tabular}

Fuente: Elaboración propia.

Cuadro 3. Estadísticos descriptivos escala BIEPS-A

\begin{tabular}{lcc}
\hline & M & DT \\
\hline Creo que sé lo que quiero hacer con mi vida. & 2,67 & 0,527 \\
Si algo me sale mal puedo aceptarlo o admitirlo. & 2,63 & 0,529 \\
Me importa pensar que haré en el futuro. & 2,57 & 0,589 \\
Puedo decir lo que pienso sin mayores problemas. & 2,26 & 0,620 \\
Generalmente caigo bien a la gente. & 2,49 & 0,554 \\
Siento que puedo lograr las metas que proponga. & 2,44 & 0,581 \\
Cuento con personas que me ayudan si lo necesito. & 2,77 & 0,459 \\
Creo que en general me llevo bien con la gente. & 2,82 & 0,398 \\
Estoy bastante conforme con mi forma de ser. & 2,51 & 0,534 \\
En general hago lo que quiero, soy poco influenciable. & 2,32 & 0,554 \\
Soy capaz de pensar en un proyecto para mi vida. & 2,72 & 0,476 \\
Puedo aceptar mis equivocaciones y tratar de mejorar. & 2,73 & 0,491 \\
Puedo tomar decisiones sin dudar mucho. & 2,17 & 0,675 \\
Encaro sin mayores problemas mis obligaciones diarias. & 2,63 & 0,558 \\
\hline
\end{tabular}

Fuente: Elaboración propia.

\subsection{Relaciones entre bienestar subjetivo y satisfacción vital}

Los resultados obtenidos muestran una correlación significativa entre bienestar subjetivo y satisfacción vital $(0,547)$, asimismo en el cuadro 4 se muestran las correlaciones significativas entre los distintos ítems de la Escala de Satisfacción Vital y la puntuación total obtenida en la Escala de Bienestar Subjetivo. Según los resultados obtenidos los aspectos de la satisfacción vital que influyen más a la hora de determinar el grado de bienestar subjetivo son: "Estoy satisfecho con mi vida" y "Hasta ahora he conseguido de la vida las cosas que considero importantes".

Con respecto a las correlaciones entre las variables de la Escala BIEPS-A y la puntuación total obtenida en la escala SWLS y a la vista del patrón correlacional, observamos que los ítems que muestran una correlación significativa a un nivel de 0,01 son: "Generalmente caigo bien a la gente", "Cuento con personas que me ayudan si lo necesito", "Creo que en general me llevo bien con la gente", "Soy capaz de pensar en un proyecto para mi vida", "Puedo tomar decisiones sin dudar mucho" y "Encaro sin mayores problemas mis obligaciones diarias".

En el cuadro 5 quedan reflejadas las correlaciones entre las dimensiones de la escala de bienestar y las distintas variables del cuestionario demográfico. Según los datos obtenidos, existe correlación significativa entre las distintas dimensiones de la escala de Bienestar y todas las variables estudiadas excepto el género. 
Cuadro 4. Correlaciones entre las variables de la SwLS y el bienestar

\begin{tabular}{|c|c|c|c|c|c|}
\hline & 1 & 2 & 3 & 4 & $\mathbf{5}$ \\
\hline \multicolumn{6}{|l|}{$\begin{array}{l}\text { 1. En la mayoría de los aspectos mi vida es como yo quiero que } \\
\text { sea }\end{array}$} \\
\hline 2. Las circunstancias de mi vida son muy buenas & \multicolumn{5}{|c|}{$0,643^{* *}$} \\
\hline 3. Estoy satisfecho con mi vida & \multicolumn{5}{|c|}{$0,742^{* *} 0,675^{* *}$} \\
\hline $\begin{array}{l}\text { 4. Hasta ahora he conseguido de la vida las cosas que } \\
\text { considero importantes }\end{array}$ & \multicolumn{5}{|c|}{$0,611^{\text {** }} 0,543^{* *} 0,676^{* *}$} \\
\hline 5. Si pudiera vivir mi vida otra vez no cambiaría casi nada & \multicolumn{5}{|c|}{$0,601^{* *} 0,456^{* *} 0,545^{* *} 0,554^{* *}$} \\
\hline Total Bienestar & \multicolumn{5}{|c|}{$0,445^{* *} 0,379^{* *} 0,512^{* *} 0,469^{* *} 0,390^{* *}$} \\
\hline
\end{tabular}

El análisis de datos reveló que las variables demográficas de edad y años de experiencia docente correlacionan de manera positiva con las dimensiones de aceptación y autonomía de la escala BIEPS-A, lo que nos indica que los participantes que puntuaron alto en estas dimensiones son capaces de tomar decisiones de manera independiente y asertiva y se sienten bien acerca de su pasado.

Cuadro 5. Correlación entre las dimensiones de la escala BIEPS-A y las variables sociodemográficas

\begin{tabular}{|c|c|c|c|c|c|c|c|c|}
\hline & 1 & 2 & 3 & 4 & 5 & 6 & 7 & 8 \\
\hline 1. Edad & & & $0,731^{* * *}$ & & $0,237^{* *}$ & $0,147^{*}$ & & \\
\hline 2. Titularidad & & & & & & $0,190^{*}$ & & \\
\hline 3. Experiencia & $0,731^{* *}$ & & & & $0,162^{*}$ & & & \\
\hline 4. Etapa & & & & & & $0,157^{*}$ & & \\
\hline 5. Aceptación & $0,237^{* *}$ & & $0,162^{*}$ & & & $0,550^{* *}$ & $0,311^{* *}$ & $0,546^{* *}$ \\
\hline 6. Autonomía & $0,147^{*}$ & $0,190^{*}$ & & $0,157^{*}$ & $0,550^{* *}$ & & $0,454^{* *}$ & $0,432^{* *}$ \\
\hline 7. Vínculos & & & & & $0,311^{* *}$ & $0,454^{* *}$ & & $0,256^{\text {*** }}$ \\
\hline 8. Proyectos & & & & & $0,546^{* * *}$ & $0,432^{* *}$ & $0,256^{* *}$ & \\
\hline
\end{tabular}

Nota: Sólo se muestran las correlaciones significativas. *** La correlación es significativa en el nivel 0,01 (bilateral). *La correlación es significativa en el nivel 0,05 (bilateral).

Fuente: Elaboración propia.

\section{Discusión y conclusiones}

El objetivo de esta investigación era explorar la influencia que determinadas variables relacionadas con el ámbito laboral del docente tienen sobre el bienestar psicológico y el grado de satisfacción de los profesionales que ejercen esta profesión. Como se comentó en el marco teórico, esta hipótesis surge del modelo interaccionista que considera que el bienestar subjetivo depende de las características del entorno y de las características personales del sujeto. Los datos empíricos sugieren que bienestar subjetivo y satisfacción vital correlacionan positivamente, lo que nos lleva a decir que aquellos docentes que se sientan satisfechos con su vida también experimentarán alto grado de bienestar subjetivo. Asimismo los resultados obtenidos en esta investigación demuestran que el bienestar psicológico de los participantes se asocia a factores como tener buenas relaciones con las personas de su entorno, contar con ayuda en caso de necesitarla, tener un proyecto vital, realizar un proceso de toma de decisiones efectivo y encarar los problemas de la vida diaria 
de manera resolutiva y que los aspectos que reflejan una mayor satisfacción vital están relacionados con la consecución de objetivos vitales y la posibilidad de cambiar algunos aspectos de su vida.

Respecto a la hipótesis de trabajo acerca de la influencia de determinadas variables demográficas en el bienestar subjetivo y la satisfacción vital del profesorado podemos concluir que la satisfacción vital correlaciona de manera significativa con la edad, la experiencia docente y la titularidad del centro, no así con el género y la etapa en la que se imparte docencia. En relación al bienestar subjetivo podemos señalar que existen correlaciones significativas con respecto al género, la edad, la titularidad del centro y los años de experiencia docente, no encontrando estas correlaciones en la etapa en la que se imparte docencia. Atendiendo a diversas investigaciones que concluyen que el género condicionaría distintas perspectivas para valorar la satisfacción y el malestar docente, sugiriendo que las mujeres presentarían una mayor tendencia hacia el agotamiento emocional a diferencia de los hombres, cuya tendencia sería hacia la despersonalización (Lau, Yuen y Chan, 2005), los resultados obtenidos en esta investigación indican que este grado de satisfacción vital y bienestar también está influido por otras variables como la edad y los años de experiencia docente.

Por otro lado, y teniendo en consideración el tamaño de la muestra, resultaría interesante ampliar el número de participantes en posteriores investigaciones a otras Comunidades Autónomas incluso a otros países, para poder establecer si existen diferencias significativas con respecto a la influencia de las variables demográficas analizadas en esta investigación.

Sigue siendo objeto de interés para futuras investigaciones estudiar la causalidad asociada a porqué determinados docentes desempeñan su labor profesional desde la motivación y el interés y otros lo hacen desde el desánimo y el agotamiento (Carrasco y Bernal, 2008; Marchesi, 2007) y cómo influyen estas actitudes en la práctica pedagógica, ya que numerosas investigaciones han concluido que los comportamientos y las actitudes del docente condicionan los resultados obtenidos por los estudiantes (Murillo, MartínezGarrido y Hernández-Castilla, 2011). Por otra parte, y basándonos en las últimas líneas de investigación relacionadas con bienestar docente, deberíamos incluir como aspecto a investigar la influencia de la inteligencia emocional en el desempeño de la labor docente y cómo la implantación de programas de educación social y emocional pueden llegar a favorecer el bienestar, resultando claves para mejorar el ejercicio de la práctica docente. Asimismo, tras la revisión de la literatura en este campo queda patente la necesidad de llevar a cabo un mayor número de investigaciones acerca del bienestar docente (De Pablos, Bravo y González, 2011; Hué, 2012), que permitan desarrollar herramientas e instrumentos innovadores y eficaces para medirlo desde una perspectiva holística y multidimensional del docente en su ámbito personal y profesional.

\section{Referencias}

Andrews, F. M. y Withey, S. B. (1976). Social indicators of well-being: Americans' perception of quality of life. Nueva York, NY: Springer. https://doi.org/10.1007/978-1-4684-2253-5

Atienza, F. L., Pons, D., Balaguer, I. y García, M. (2000). Propiedades psicométricas de la escala de satisfacción con la vida en adolescentes. Psicothema, 12(2), 1-19. 
Ballesteros, B. P., Medina, A. y Caycedo, C. (2006). El bienestar psicológico definido por asistentes a un servicio de consulta psicológica en Bogotá, Colombia. Universitas Psychologica, 5(2), 239-258.

Campbell, A., Converse, P. y Rodgers, W. (1976). The quality of American life: Perceptions, evaluations and satisfactions. Nueva York, NY: Russell Sage Foundation.

Carrasco, J. G. y Bernal, A. (2008). Institución y decepción. La salubridad institucional y la práctica docente. Revista Española de Pedagogía, 66(241), 405-423.

Casas, F., Rosich, M. y Alsinet, C. (2000). El bienestar psicológico de los preadolescentes. Anuario de Psicología, 31(2), 73-86.

Casullo, M. M. y Brenlla, M. E. (2002). Evaluación del bienestar psicológico en Iberoamérica. Buenos Aires: Paidós.

Casullo, M. M. y Solano, A. C. (2000). Evaluación del bienestar psicológico en estudiantes adolescentes argentinos. Revista de Psicología, 18(1), 35-68.

Chávez, R. C. (2009). Condiciones de trabajo y bienestar/malestar docente en profesores de enseñanza media de Santiago de Chile. Educação \& Sociedade, 30(107), 409-426. https://doi.org/10.1590/S0101-73302009000200006

Cornejo, R. y Quiñónez, M. (2007). Factores asociados al malestar/bienestar docente. Una investigación actual. REICE. Revista Iberoamericana sobre Calidad, Eficacia y Cambio en Educación, 5(2), 75-80.

Csikszentmihalyi, M. y Seligman, M. E. (2000). Positive psychology: An introduction. American Psychologist, 55(1), 5-14. https://doi.org/10.1037/0003-066X.55.1.5

De Pablos, J. M., Bravo, P. S. C. y González, M. T. (2011). Bienestar docente e innovación con tecnologías de la información y la comunicación. Revista de Investigación Educativa, 29(1), 5981 .

Díaz, D., Rodríguez-Carvajal, R., Blanco, A., Moreno-Jiménez, B., Gallardo, I., Valle, C. y Dierendonck, D. (2006). Adaptación española de las escalas de bienestar psicológico de Ryff. Psicothema, 18(3), 572-577.

Diener, E. y Seligman, M. E. (2004). Beyond money: Toward an economy of well-being. Psychological Science in the Public Interest, 5(1), 1-31. https://doi.org/10.1111/j.09637214.2004.00501001.x

Diener, E. D., Emmons, R. A., Larsen, R. J. y Griffin, S. (1985). The satisfaction with life scale. $\begin{array}{llll}\text { Journal of Personality } & \text { Assessment, }\end{array}$ https://doi.org/10.1207/s15327752jpa4901_13

Diener, E. y Ryan, K. (2009). Subjective well-being: A general overview. South African Journal of Psychology, 39(4), 391-406. https://doi.org/10.1177/008124630903900402

Domínguez, S. A. (2014). Análisis psicométrico de la escala de bienestar psicológico para adultos en estudiantes universitarios de Lima: Un enfoque de ecuaciones estructurales. Psychologia: Avances de la Disciplina, 8(1), 23-31. https://doi.org/10.21500/19002386.1211

Eid, M. y Larsen, R. J. (2008). The science of subjective well-being. Londres: Guilford Press.

Extremera, N., Salguero, J. M. y Fernández-Berrocal, P. (2011). Trait meta-mood and subjective happiness: A 7-week prospective study. Journal of Happiness Studies, 12(3), 509-517. https://doi.org/10.1007/s10902-010-9233-7 
Harris, P. R. y Lightsey, O. R. (2005). Constructive thinking as a mediator of the relationship between extraversion, neuroticism, and subjective well-being. European Journal of Personality, 19(5), 409-426. https://doi.org/10.1002/per.544

Hué, C. (2012). Bienestar docente y pensamiento emocional. Revista Fuentes, 12, 47-68.

Hulin, C. L. y Judge, T. A. (2003). Job attitudes. En I. Weiner, Handbook of psychology (pp. 234-254). Nueva York, NY: Wiley. https://doi.org/10.1002/0471264385.wei1211

Karasek, R. A. y Theorell, T. (1990). Health work. Nueva York, NY: Basic Book.

Keyes, C. L., Shmotkin, D. y Ryff, C. D. (2002). Optimizing well-being: The empirical encounter of two traditions. Journal of Personality and Social Psychology, 82(6), 1007-1022. https://doi.org/10.1037/0022-3514.82.6.1007

Lau, P. S., Yuen, M. T. y Chan, R. M. (2005). Do demographic characteristics make a difference to burnout among Hong Kong secondary school teachers? En D. Shek, Y. Chan y P. Lee (Eds.), Quality-of-life research in chinese, western and global contexts (pp. 491-516). Ámsterdam: Springer. https://doi.org/10.1007/1-4020-3602-7_17

Marchesi, A. (2007). Sobre el bienestar de los docentes. Madrid: Alianza Editorial.

Maslach, C. (2003). Job burnout new directions in research and intervention. Current Directions in Psychological Science, 12(5), 189-192. https://doi.org/10.1111/1467-8721.01258

Murillo, F. J., Martínez-Garrido, C. y Hernández-Castilla, R. (2011). Decálogo para una enseñanza eficaz. REICE. Revista Iberoamericana sobre Calidad, Eficacia y Cambio en Educación, 9(1), 627.

Pavot, W. y Diener, E. (1993). Review of the satisfaction with life scale. Psychological Assessment, 5(2), 164-167. https://doi.org/10.1037/1040-3590.5.2.164

Ryan, R. M. y Deci, E. L. (2001). On happiness and human potentials: A review of research on hedonic and eudaimonic well-being. Annual Review of Psychology, 52(1), 141-166. https://doi.org/10.1146/annurev.psych.52.1.141

Ryff, C. D. (1989). Happiness is everything, or is it? Explorations on the meaning of psychological well-being. Journal of Personality and Social Psychology, 57(6), 1069-1084. https://doi.org/10.1037/0022-3514.57.6.1069

Ryff, C. D. y Singer, B. (1996). Psychological well-being: Meaning, measurement, and implications for psychotherapy research. Psychotherapy and Psychosomatics, 65(1), 14-23. https://doi.org/10.1159/000289026

Seligman, M. E. (2011). Learned optimism: How to change your mind and your life. Nueva York, NY: Vintage.

Seligson, J. L., Huebner, E. S. y Valois, R. F. (2003). Preliminary validation of the brief multidimensional students' life satisfaction scale (BMSLSS). Social Indicators Research, 61(2), 121-145. https://doi.org/10.1023/A:1021326822957

Vázquez, C., Hervás, G., Rahona, J. J. y Gómez, D. (2009). Bienestar psicológico y salud: Aportaciones desde la psicología positiva. Anuario de Psicología Clínica y de la Salud, 5, 1528.

Veenhoven, R. (1991). Is happiness relative? Social Indicators Research, 24(1), 1-34. https://doi.org/10.1007/BFo0292648

Verhoeven, R., Kraaij, V., Joekes, K. y Maes, S. (2003). Job conditions and wellness/health outcomes in Dutch secondary school teachers. Psychology and Health, 18(4), 473-487. https://doi.org/10.1080/0887044031000147201 


\section{Breve CV de los autores}

\section{Eva María Muñoz Campos}

Licenciada en Psicología Clínica y del Trabajo por la Universidad Complutense de Madrid. Doctoranda en Ciencias de la Educación en la Universidad Autónoma de Madrid, Facultad de Formación de Profesorado y Educación, en el Departamento de Didáctica y Teoría de la Educación. Profesora asociada en el Dpto. de Psicología Evolutiva y de la Educación en la Facultad de Formación de Profesorado y Educación de la Universidad Autónoma de Madrid. Desde 2008 desarrolla su labor profesional como docente de C.F.G.S. en la Familia Profesional de Servicios Socioculturales y a la Comunidad y en EOEPS de la Comunidad de Madrid. Forma parte del grupo de investigación "Cambio Educativo para la Justicia Social” (GICE) de la UAM desde el año 2017. ORCID ID: 0000oo01-6146-1036. Email: eva.munnoz@uam.es

\section{Antonio Fernández González}

Doctor por la Universidad Autónoma de Madrid, Diplomado en Magisterio por la Universidad de Santiago de Compostela, Licenciado en Pedagogía y Psicopedagogía por la Universidad Complutense de Madrid. Profesor asociado en la Universidad Autónoma de Madrid y en la Consejería de Educación de la Comunidad de Madrid. Miembro del grupo de investigación GICE y del consejo de redacción de RIEJS "Revista Internacional de Educación para la Justicia Social”. Es coautor del test TESIS (Test de Sensibilidad a las Interacciones Sociales) publicado en Albor-Cohs. ORCID ID: 0000-0001-8603-7979. Email: toni.fernandez@uam.es

\section{Liliana Jacott}

Mi experiencia investigadora se centra, principalmente, en el ámbito de la Psicología del Desarrollo y más específicamente en el ámbito de la Ciudadanía, el Desarrollo Humano y la Justicia Social. Actualmente soy la coordinadora IP de la línea de investigación sobre Desarrollo Humano y Educación, dentro del Grupo de investigación GICE (Cambio educativo para la Justicia Social) de la UAM. He participado en distintos proyectos de investigación competitivos a nivel nacional e internacional. El resultado de estas investigaciones aparece recogido en diferentes artículos de investigación publicados tanto en prestigiosas revistas internacionales tales como: Citizenship Teaching and Learning, Human Rights Review, European Journal of Educational Research y Revista Internacional de Educación para la Justicia Social, así como en capítulos de libros internacionales publicados por prestigiosas editoriales tales como en CiCe, London Metropolitan University y presentaciones en Congresos nacionales e internacionales. He sido miembro de la Red Europea Jean Monnet CiCe "Children's Identity and Citizenship European Association” (desde 2009), participando como experta internacional en el Comité Internacional de la Summer Doctoral Research Student Conference desde 2015. ORCID ID: 0000-0002-5826-3693. Email: liliana.jacott@uam.es 